\title{
Entrelacs
}

Cinéma et audiovisuel

6 | 2007

L'Arbre

\section{Arbre abattu, arbre redressé}

Le pin de I Dimenticati (Les Oubliés), de Vittorio de Seta (1959)

François de la Bretèque

\section{(2) OpenEdition}

Journals

Édition électronique

URL : http://journals.openedition.org/entrelacs/167

DOI : 10.4000/entrelacs. 167

ISSN : 2261-5482

Éditeur

Éditions Téraèdre

Édition imprimée

Date de publication : 1 mars 2007

Pagination : 10-22

ISBN : 978-2-912868-70-1

ISSN : 1266-7188

Référence électronique

François de la Bretèque, "Arbre abattu, arbre redressé », Entrelacs [En ligne], 6 | 2007, mis en ligne le 01 août 2012, consulté le 01 mai 2019. URL : http://journals.openedition.org/entrelacs/167 ; DOI :

10.4000/entrelacs. 167

Ce document a été généré automatiquement le 1 mai 2019.

Tous droits réservés 


\title{
Arbre abattu, arbre redressé
}

\author{
Le pin de I Dimenticati (Les Oubliés), de Vittorio de Seta (1959)
}

\author{
François de la Bretèque
}

1 Un pin géant est le sujet et la figure centrale du film de Vittorio De Seta I Dimenticati, mais c'est un arbre que l'on abat, sacrifié, ensuite transporté et redressé au cœur des habitations des hommes.

2 Nous sommes à Alessandria del Carretto, village de Calabre. Fin des années 50. L'Italie qui se reconstruit, celle du miracle économique, n'est pas arrivée jusqu'ici. C'est un complet isolat économique, culturel et humain. La route qui devait le relier au monde extérieur s'interrompt brutalement. Les travaux ont été arrêtés pour une raison inconnue et ne semblent pas devoir reprendre de sitôt. Le village n'est ravitaillé qu'à dos de mulets. Cependant, nous dit le commentaire, une fois l'an, la fête du printemps fait revivre la communauté autour d'un cérémonial qui remonte à la nuit des temps. On va chercher dans la montagne, bien plus haut, un pin gigantesque que l'on abat, que l'on redescend jusqu'au village à la traîne, et que l'on redresse au centre de la place après une bénédiction religieuse. Les hommes les plus hardis s'essaient à y grimper pour récupérer les offrandes qu'on a accrochées à sa cime. La fête se conclut par un banquet collectif, dernière manifestation, nous dit encore le commentaire, d'une communauté oubliée que peut-être la route sortira de son isolement, faisant du même coup disparaître cette coutume ancestrale.

3 Vittorio De Seta connaît bien la Calabre, il en est originaire et il y a tourné plusieurs de ses premiers films. De 1954 à 1959, cet ancien étudiant en architecture passionné de photographie a réalisé en indépendant presque complet et avec des moyens réduits dix courts métrages qui constituent un massif singulier dans l'histoire du cinéma italien postnéo-réaliste. Il s'est acheté une caméra $35 \mathrm{~mm}$. après avoir été assistant sur un film que Jean-Paul Le Chanois tournait en Sicile. Il décide de filmer ceux qu'il côtoie et que le monde ignore. Ces dix films « documentaires ", tous tournés dans le grand Mezzogiorno (Sicile, Sardaigne et Calabre), ont pour sujet les travaux et les jours de ses habitants les plus humbles et les transformations de leur environnement. Tous sont conçus selon la même méthode : s'immerger dans le terrain que l'on va filmer, respecter une distance par 
rapport aux gens que l'on enregistre, s'accorder le temps nécessaire pour voir vivre ces gens. De Seta compose ses plans comme un photographe son cadre. Il travaille beaucoup son montage. Il ajoute une bande sonore composée de bruits enregistrés sur place et de chants traditionnels, livrant les images sans commentaire explicatif surplombant. Quelques textes apparaissant sur l'écran se contentent de situer lieux et gestes. Dans I Dimenticati, pour la première et seule fois, cette fonction est dévolue à une voix (la sienne) qui n'intervient que trois fois. On a pu redécouvrir cette partie de son œuvre grâce à la Filmoteca Regionale Siciliana de Palerme qui les a récemment restaurées.

4 Comme l'ensemble de l'œuvre « documentaire » de De Seta (Bandits à Orgosolo inclus), I Dimenticati est un film magnifique qui décourage l'exégèse tant par la discrétion absolue $\mathrm{du}$ discours que par sa splendeur formelle, qu'il faut bien se garder de dénigrer comme l'ont fait certains historiens soucieux de le dévaluer à l'aune d'une certaine idée qu'ils se font du néoréalisme ${ }^{1}$. De Seta ne se revendique pas de ce courant, il a tourné en complet indépendant, même s'il est évident qu'il avait vu et connaissait beaucoup de films et notamment ceux de ses compatriotes transalpins. Il s'est ainsi expliqué sur son style : «ce qui me gêne dans beaucoup de films actuels, c'est le style que les cinéastes ont voulu donner. On ne fait pas assez confiance aux capacités du public. Ou bien il y a une recherche artistique additionnelle, comme pour dire : je vous donne ça en plus pour moi, le style est dicté par la nature de ce que je veux dire. (...) si malgré tout il y a un style, c'est involontaire. $»^{2}$

5 L'arbre, je l'ai dit, constitue le centre, l'axe (le mot s'impose) de ce film. Mais c'est un arbre que l'on abat. Ce geste sacrilège que pratiquent généralement sans états d'âme les Méditerranéens, que le développement du tourisme, de l'urbanisation, des transports, nous a habitués à voir tout autour de nos villes et de nos campagnes, n'est ici l'objet d'aucune indignation : c'est qu'il s'agit d'un geste sacré (l'inverse du sacrilège) que

6 l'on nous donne à voir, sans chercher à l'interpréter ni à porter de jugement. Ici, on ne coupe l'arbre que parce qu'il y a une raison supérieure; on le respecte, on s'excuserait presque auprès de lui, comme le font certains chasseurs chez Rouch ou chez Flaherty.

7 On pourrait prendre I Dimenticati comme un document ethnographique, dans la mesure où il enregistre et donne à connaître une coutume en voie de disparition d'une population rurale de l'Europe contemporaine. Cependant, la posture de De Seta n'est pas celle de l'ethnologue: ni celle de l'ethnologue traditionnel qui livrerait un savoir savant sur ce qu'il montre, ni celle de l' « ethnologie participative » d'un Jean Rouch - qui, coïncidence à noter, commence ses tournages expérimentaux à peu près à la même date, en isolé complet lui aussi.

8 Le film se prêterait aussi à l'exégèse politique, quoique ce ne soit en aucune façon son propos affiché. Il y a certes des responsables pour que les habitants d'Alessandria del Carretto soient ainsi « oubliés », mais on ne nous les désigne pas.

9 Ce qui donne leur vraie dimension aux films de De Seta, c'est leur dimension poétique. J'entends par là l'appel à un arrière monde, l'invitation à voir, au-delà des activités présentes, leur enracinement profond dans le temps humain, l'anoblissement des pratiques les plus humbles de ces paysans calabrais par ce qui les rattache aux mythes et aux croyances les plus élevées de la culture occidentale. C'est cela qui fait qu'un film comme celui-ci nous parle encore, nous citadins d'un pays développé du début du XXI siècle. 
L'attention aux gestes et aux outils, voilà la première caractéristique qui frappe chez De Seta. Pour abattre l'arbre, le traîner jusqu'au village et le redresser, les autochtones ont recours à des techniques ancestrales dont certaines doivent remonter à la préhistoire. Il est scié avec une scie de long. Il est ébranché et écorcé avec un soin méticuleux. Cette mise à nu a déjà un côté rituel. On fabrique ensuite de solides liens à l'aide de tiges de bois que l'on tord au feu et qui serviront à tirer l'énorme tronc. On fait progresser ce dernier sur des rouleaux, à la façon des populations néolithiques. Tout cela à la seule force des bras : l'exercice apparaît fort dangereux sur ces pentes caillouteuses. Le village entier participe à l'opération : les enfants tournent autour du cortège, lâchent des pétards ; les femmes sont montées à la montagne pour préparer les collations de leurs hommes. Des gestes de mains anonymes filmées en gros plan jusqu'aux plans larges qui montrent tout le groupe, le sens se construit clairement. La solidarité et l'effort collectif sont les solutions imposées pour que l'homme l'emporte dans ce combat inégal contre les forces naturelles. Comme l'a écrit Gian Piero Brunetta : «dans ces documentaires, la nature comme dans l'Homme d'Aran et dans tout le cinéma de Flaherty - a encore la supériorité et l'homme éprouve encore le même sentiment de terreur panique dans sa confrontation avec le déchaînement des forces naturelles et des mystères de la nuit et du feu que son ancêtre préhistorique $»^{3}$.

11 Mais la survie élémentaire ne se sépare pas d'un sentiment religieux. Le lendemain de la descente, la journée commence par une cérémonie : des pétards éclatent dans le ciel. on sort de l'église le buste d'un saint (un saint pape, apparemment) pour une procession, et l'on fait bénir des pains, des fruits, des rameaux garnis de friandises très semblables à ceux que l'on donne aux enfants le dimanche des Rameaux encore aujourd'hui dans le Midi et en Catalogne. Ils font l'objet d'une mise aux enchères dont on ne comprend pas bien la fonction. Ces fruits de la terre serviront d'offrandes que l'on accrochera aux branches sommitales de l'arbre. Celles-ci ont été conservées, et les hommes vont les rattacher au sommet du tronc avec une technique ingénieuse et compliquée dans laquelle n'entrent encore que des outils traditionnels.

12 Le pin, dans la mythologie gréco-romaine, est l'arbre de Cybèle (et son fruit l'emblème de Dionysos, mais il ne joue ici aucun rôle). Tous les mythes qui se rattachent à lui dans l'aire méditerranéenne en font un symbole d'immortalité. Voici une description des fêtes de Cybèle à Rome :

"Un pin était abattu et transporté dans le temple du Palatin par une confrérie qui devait à cette fonction son nom de dendrophores. Ce pin, enveloppé comme un cadavre de bandelettes de laine et enguirlandé de violettes figurait Attis mort (l'époux de la déesse) (...) le lendemain était un jour de tristesse où les fidèles jeûnaient et se lamentaient auprès du corps du dieu... Veillée mystérieuse... résurrection attendue ... on passait alors des cris de désespoir à une jubilation délirante... Avec le renouveau de la nature, Attis s'éveillait de son long sommeil et, en des réjouissances déréglées, des banquets plantureux, on donnait libre cours à la joie provoquée par son retour à la vie $»^{4}$.

13 Ne nous laissons pas abuser par des ressemblances pourtant troublantes. Les Calabrais de 1950 ne rejouent évidemment pas les mystères de Cybèle. Les folkloristes nous incitent à nous méfier des spéculations sur les survivances d'une aussi longue durée ${ }^{5}$. Ce qui compte en effet ici est l'esprit qui enveloppe la cérémonie. L'anthropomorphisation de l'arbre, le tronc vu comme corps nu, la ferveur qui l'entoure, le dérèglement qui accompagne sa " résurrection " (ici représentée par son érection), tout cela dégage incontestablement une atmosphère païenne qui est d'ailleurs le sort de bien des cérémonies populaires en 
Italie et en terre catholique en général. Il est d'ailleurs à remarquer que le pin ne joue pas de rôle significatif dans la symbolique biblique et chrétienne.

La fête de printemps de ce village de Calabre se rattache à un rite calendaire lié à la renaissance de la végétation Les ethnologues nous diraient que le rituel des «mais » est répandu dans toute l'Europe, «ces grands arbres que l'on plante au milieu du village ou sur la place du marché et qui le sont avec la participation, non pas seulement des jeunes gens, mais avec celle de la population toute entière. L'unanimité des paysans est ici nécessaire pour aller chercher cérémoniellement le mât dans la forêt (....) L[a] fonction [de ces mais] semble de nature magique. Le groupe de la Jeunesse masculine, plantant au milieu de la communauté villageoise un arbre qu'ils sont allés chercher dans une forêt (lieu symbolisant la nature) suivant des droits établis, s'assure de cette manière d'une emprise sur la végétation renaissante afin de pouvoir contrôler la croissance dans les pratiques agricoles ». On nous précise même que «le plus souvent, ce mai collectif est un bouleau ou un sapin $»^{6}$. Il faut noter en passant que De Seta n'indique ni la date de la fête ni le nom du saint, voulant ainsi éviter toute interprétation qui se réduirait à de l'ethnologie religieuse.

15 Descendu sur la place, le pin est planté au centre et transformé en mât. Mais on a pris soin de lui conserver son apparence d'arbre (ce qui le différencie d'un simple mât « de Cocagne »). Seules ont été conservées quelques ramures sommitales rattachées au tronc principal par un système en baïonnette. Ainsi recomposé, il ressemble à un dessin d'enfant ou à une illustration médiévale. Une idée d'arbre, plutôt qu'un arbre singulier. Il relie la terre au ciel, ce ciel que nous a montré le premier plan de la séquence (la fumée des pétards s'y détachait à contre-jour). Il retrouve ainsi sa fonction archétypale d'axis mundi? .

16 L'homme va devoir y monter et se mesurer à lui. On ne lui facilite pas la tâche : le tronc est lisse, les candidats à l'ascension conservent leurs chaussettes et un organisateur vérifie même qu'ils n'y ont rien dissimulé. C'est à la seule force des cuisses et des bras que doit se faire l'ascension. Mais il ne faut pas se tromper: l'exercice n'a rien d'une compétition sportive. Il s'agit, on l'a compris, d'une ascension symbolique. Il n'y a d'ailleurs pas de vainqueur désigné, l'impétrant qui réussit l'épreuve après un camarade qui n'y est pas parvenu rentre dans la foule sitôt qu'il a regagné le sol. Du haut du mât, il a lancé à la foule quelques-uns des prix qu'il a décrochés et semble ne rien avoir gardé pour lui. Il est en fait le porte parole de la communauté et sa victoire est celle de l'ingéniosité humaine.

La caméra de De Seta, comme l'a noté Brunetta, est utilisée comme «l'instrument d'enregistrement d'une voix collective ». Elle ne donne la vedette à personne. Sauf à l'arbre, dira-t-on, mais l'arbre représente iconographiquement l'unité de la communauté humaine. En bas, le peuple; en haut, un délégué de celui-ci, un observateur. Une image fort semblable se rencontre dans Le Temps de l'espadon : un guetteur est placé sur un mât planté au milieu du bateau. La caméra l'observe et prend même occasionnellement sa place (au prix d'une acrobatie technique impossible à détecter) et montre, par ses yeux, la surface liquide sous laquelle passent les poissons ainsi que ses camarades restés sur le pont du bateau. De Seta recourt parfois ainsi aux grandes plongées mais jamais gratuitement. Le point de vue est toujours celui d'un observateur potentiel. Son regard ne quitte pas la position du groupe. 
18 Mais ces plans qui embrassent un espace très large ont une autre fonction, celle d'intégrer l'homme dans le cosmos. Les éléments naturels sont très présents dans I Dimenticati comme dans les autres films : le brouillard enveloppe le village à l'arrivée de la caravane de mulets; des nuages lourds planent au-dessus des haleurs du tronc; sans parler des torrents, des rochers, des éboulis qui cernent le hameau. Pour les rendre, la photographie adopte un picturalisme évident qui se nourrit de toute la tradition de la grande peinture italienne de l'ère baroque, avec des clairs-obscurs fortement expressifs. Les nuages, le ciel, l'eau y sont traités « a tempera », comme sur une toile. Les couleurs irréalistes du Ferraniacolor vont dans le sens de cette théâtralisation.

19 I Dimenticati, à la différence des films qui l'ont précédé, n'est pas tourné en écran large. Néanmoins De Seta y conserve le principe de composition qu'on y trouvait. Les motifs se répètent sur la surface de l'image: hommes ou femmes debout, figures de procession, objets ou animaux (voir par exemple la caravane de mulets ou le cortège des hommes tirant le tronc) créent un rythme plastique spécifique qui va dans le sens du propos: l'effort collectif imposé pour survivre. C'est à l'épopée dans ses formes les plus antiques que ce rythme, fait de « versets » successifs enchaînés avec une certaine lenteur, ne peut manquer de faire penser. «Ces fragments résiduels de civilisation archaïque ont trouvé en de Seta un chantre capable de retrouver des tonalités épiques qui use de la machine de prise de vues comme d'un instrument d'enregistrement d'une voix collective » dit fort justement G. P. Brunetta ${ }^{8}$. De son côté, Pierre Leprohon avait noté à propos du premier long métrage de De Seta qui suivra I Dimentacati : "Bandits à Orgosolo, ainsi que Salvatore Giuliano, renoue avec un certain côté épique du néo-réalisme, celui de la marche à la mort du Soleil se lèvera encore, celui de De Santis et de Lattuada .» ${ }^{9}$ Marche à la mort ? Celle d'une humanité abandonnée à elle-même, celle d'une culture condamnée à disparaitre, comme le suggère le commentaire final.

20 Tout le film se structure autour de la coupure et de la réconciliation (autre façon de signifier la mort et la vie), que symbolisent ici l'horizontal et le vertical. A la route coupée (horizontal) répond ainsi l'arbre coupé que l'on redresse (vertical). La première contraint les villageois à une peine qu'ils n'ont pas choisie : transporter à dos d'homme et de mulet les biens nécessaires pour survivre par un sentier raide et accidenté qui traverse des ruisseaux en crue. Par la route coupée s'écoule une vie qui se tarit lentement Le second (le pin) est l'objet d'une peine consentie. Rien ni personne n'oblige les villageois à se donner tout ce mal, si ce n'est qu'il redonne un sens à leur travail : les intégrer au rythme du cosmos, célébrer le rassemblement de leur communauté.

21 De Seta a-t-il simplement voulu fixer une coutume archaïque avant qu'elle disparaisse, cédant au réflexe de "la beauté du mort» dont parle Michel de Certeau? A-t-il voulu dénoncer l'oppression de populations arriérées par des coutumes d'un autre âge ? Il n'est pas interdit d'interpréter le film dans ce sens, mais sa vraie signification ne se trouve pas là. Quand sur la place du village le grand pin se redresse, qu'il ombrage la population fervente rassemblée à ses pieds, quand le paysan aux mains et aux pieds nus en redescend après avoir touché son sommet, c'est l'homme lui-même, la dignité humaine, qui se remet debout sous le ciel éternel. 


\section{NOTES}

1. Par exemple Roger Boussinot dans son Encyclopédie du cinéma, tome I A-H, Bordas 1980.

2. Cité par Guy Gauthier, Le Documentaire, un autre cinéma, Nathan Université 1995 p. 205, citant lui-même Aldo Tassone, La Revue du cinéma - Image et son, n² 298, sept. 1975.

3. Gian-Piero Brunetta, Cent'anni di cinema italiano, Roma, Laterza, 1995, t. 2 p. 256, ma traduction.

4. Beaujeu, Defradas, Le Bonniec, Les Grecs et les Romains, Paris 1967, cité par, J. Chevallier et A. Gheerbrandt, Dictionnaire des symboles, Robert Laffont 1969 p. 608.

5. Nicole Belmont s'appuyant sur Arnold Van Gennep écrit : « il est indéniable que le Haut Moyen Age a constitué une charnière historique pour le folklore. La France [je pense que l'on pourrait en dire autant de l'Italie] était alors totalement imprégnée de christianisme et c'est probablement à partir de la liturgie et des dogmes chrétiens que se sont créés ces bourgeons adventices que sont les croyances et les coutumes populaires »: Mythes et croyances dans l'ancienne France, Flammarion, questions d'histoire, 1973, p. 65. Pour ma part, je parlerais de "refloklorisation », comme disent les médiévistes : voir par exemple Daniel Poirion, Le Merveilleux dans la littérature française du Moyen Age, PUF 1982 et Jacques Le Goff, Le Merveilleux dans l'Occident médiéval, 1978, repris dans Un Autre Moyen Age, Gallimard Quarto 1998.

6. Nicole Belmont, Mythes et croyances dans l'ancienne France, Flammarion 1973 p. 93 ; voir ibid. p. 168 la description par Van Gennep d'un mai dans la région niçoise qui se rapproche beaucoup de celui de Alessandria del Carretto.

7. Gérard de Champeaux, dom Sébastien Sterck, Le Monde des symboles, Zodiaque, 1966, pp. 297-323.

8. Ibid, Brunetta p. 256, ma traduction

9. Pierre Leprohon, le Cinéma italien, Seghers, 1966, p. 202.

\section{AUTEUR}

\section{FRANÇOIS DE LA BRETÈQUE}

Maître de Conférences, Université Paul Valéry Montpellier III 